\title{
Modelling dynamic failure of concrete with meshfree methods
}

\author{
Rabczuk T. Eibl J. \\ Institute of Concrete Structures and Building Materials, University of Karlsruhe, \\ 76128 Karlsruhe, Germany
}

\begin{abstract}
This paper studies the dynamic failure of concrete structures under blast and impact loading. We will propose a model which can capture many important effects when concrete is subjected to high dynamic loading conditions and large deformations. These effects are the strength increase which we attribute to inertia effects, the compaction of the material under high hydrostatic pressure and the anisotropy of the material in tension. A Lagrangian particle method is used which can easily handle large deformations and fracture. Fragmentation occurs naturally governed by the constitutive model due to separation of the particles. Comparisons between experiments and the simulations are discussed and show good agreement.
\end{abstract}

Key words: meshfree particle methods; dynamic fracture; concrete; SPH; strain rate

\section{INTRODUCTION}

Dynamic failure of solids is of interest in many application, e.g. in military applications such as fragmentation of bombs or resistance of bunkers but also in civil applications such as demolition of buildings by blasting or containers under high internal pressure. We are mainly interested in the failure or fracture of concrete structures under high dynamic loading conditions such as in blasts or high velocity impacts.

Dynamic fracture is characterized by the initiation, propagation and intersection of millions or billions of cracks. The failure pattern of structures under dynamic loading is therefore very different to the failure pattern of structures

Email address: t-rabczuk@northwestern.edu (Rabczuk T.). 
subjected to static loading. In the latter case, the specimen will exhibit very few cracks and large fragments while in the first case, the specimen will have many cracks and many small fragments.

Studies from e.g. $[2,9,25,40,50]$ have shown that some of the main parameters which influence dynamic failure or fragmentation are the load amplitude, the load velocity, the crack speed and the heterogeneity of the material. The load amplitude is usually closely related with the load velocity, so in practice it is difficult to produce static load amplitudes much larger than the material strength since the structure fails when the static material strength is exceeded. Under high dynamic loading conditions such as in an explosive or impact event, the load amplitude can be a multiple of the material strength. High hydrostatic pressures at the location where the load is applied were measured and often a pulverization of the material is observed, see e.g. [1,40]. For many solids, large strength increases were observed. Note that the load velocity and the crack speed are not proportional. The crack speed and hence crack intersections determine mainly the size of the fragments while the load velocity governs the crack initiation. The crack speed generally cannot exceed the theoretical maximum wave speed, i.e. the Rayleigh wave speed. However, we know from experiments (see e.g. [9]), that the crack speed is by far smaller than the Rayleigh wave speed. Eibl et al. [15,16] attribute this effect to inertia effects of the masses of the crack edges at crack opening, at least for dynamic loadings, see also Rossi [50].

It is also well known that homogenous materials such as steel exhibit more cracks and finer fragments compared to heterogenous materials such as concrete. In homogenous materials the deviation of the material strength at different material points is rather small so that cracks are initiated at many material points simultaneously if a uniform load is applied such as in containers under internal pressure. Heterogenous materials have a larger deviation of the material strength at different material points and hence less cracks are initiated. This effect diminishes with increasing load amplitude and velocity.

The initiation and evolution of millions of cracks complicate the numerical analysis of dynamic failure since it becomes from a computational point of view impossible to model every single crack and crack interactions which form the fragments. Hence, mainly phenomenological models are employed which relate the initiation and propagation of cracks to some empirical internal variables. The key point for a successful and reliable prediction of dynamic failure in a numerical analysis is the constitutive model. We will propose a model which includes (in our opinion) the most important effects for concrete structures under high dynamic loading conditions, i.e. strain rate effects depending on the load history, compaction of the material under high hydrostatic pressure and the anisotropy of concrete in tension. 
Large deformations in a numerical analysis can cause difficulties. The numerical tool itself becomes important. Lagrangian finite elements have problems to handle large deformations because of severe mesh distortions which can result in singular Jacobi matrices which leads to the termination of the computation. Remeshing becomes necessary. Data has to be mapped to the new mesh which leads to loss of accuracy. We have used a Lagrangian meshfree particle method to simulate the dynamic failure or fragmentation of concrete. Meshfree methods have advantages over Lagrangian finite elements since they can easily handle large deformations and separation of particles.

The article is arranged as follows: In the next section, we will classify theories for dynamic fracture based on numerical analyses and review some approaches. Then we propose our constitutive model. In section 3.2, we will briefly describe the meshfree method. Finally, we will apply our method to concrete under blast and impact loading. The results are compared to experimental data and show good agreement.

\section{DYNAMIC FRACTURE MODELS}

One of the first fragmentation theories was invented by Mott [39] in 1943. The main assumption was an exponential distribution of the strain due to failure along the circumference of the cylinder. He pointed out that the cylinder would fragment into an infinite number of fragments if the strain due to failure is identical for all material points. Hence the standard deviation of the strain due to failure determines mainly the fragment size. This matches well with observations that homogenous materials exhibit smaller fragments than heterogenous materials. Moreover, he assumes that an unloading zone propagates around the crack where no further cracks can be initiated. Therefore, the propagation velocity of the unloading zone also determines the fragment size.

Dynamic failure theories can be classified into two groups. The first group are mechanical statistical theories. This group can be classified into further two groups. These groups describe the fragmentation process either on macroscopic or microscopic level. More sophisticated (multi-scale) models based on the molecular or atomistic structure exist (see e.g. [26,49]) and become more and more popular, but at the moment they are limited to small amount of cracks or dislocations. Most multi-scale models are also restricted to materials with crystallin structure and are therefore not suitable for materials as concrete. On the other hand, theories based on energy principles were developed.

Micro-mechanical statistical theories were pursued e.g. by Curran et al. [1012] and implemented in a Lagrangian finite element code. To finally obtain 
fragment sizes, the microscopic crack or void radius obtained from the constitutive model is related to the radius of the fragments. Also the number of fragments are related to the number of cracks. As they pointed out, this theory is only applicable if the maximum fragment size is much smaller than the element size. Another alternative is to model the microstructure of the material directly. This idea was pursued e.g. by Morikawa et al. [38], Donze et al. [14] and Cundall [8] using discrete elements. One difficulty in this approach is to find suitable models so that the macroscopic behavior is reproduced appropriately.

Macroscopic dynamic failure theories based on phenomenological continuum models were pursued e.g. by Grady and Kipp [19-21]. In these models, an average fragment diameter is usually related to variables such as strain rate, some internal variables (often damage) or the stress intensity factor $K_{I}$.

Aimone et al. [1] studied the fragmentation of rock subjected to impact loading. They observed that the fragments contain many cracks. In figure 1, some of their results are shown. At an impulse of $2.5 \mathrm{GPa}$ and an impulse duration of $1 \mu \mathrm{s}$, we can read a crack density $S_{v}$ of $120 \mathrm{~cm}^{2} / \mathrm{cm}^{3}$ from figure $1 \mathrm{~b}$. The mean particle or fragment diameter for this case is $9 \mathrm{~cm}$, see figure 1a. Assuming spherical fragments and that the fragments do not contain internal cracks, the crack density can be computed as the relation of the surface of the sphere to the volume of the sphere which is $d / 6$ when $d$ is the fragment diameter. For our case, we obtain a crack density of $1.5 \mathrm{~cm}^{2} / \mathrm{cm}^{3}$ which is by far smaller than $120 \mathrm{~cm}^{2} / \mathrm{cm}^{3}$. Hence, the internal damage is not a suitable measurement for the fragment size.

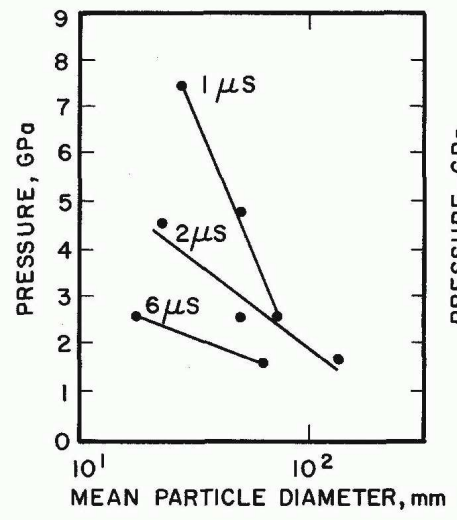

(a)

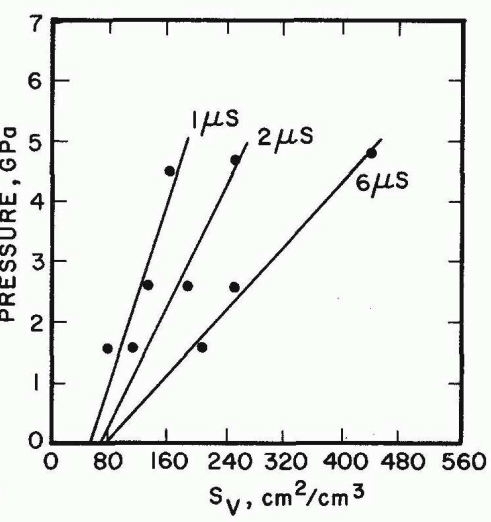

(b)

Fig. 1. Fragmentation of rock (Aimone et al. [1]) a) Pressure versus mean particle diameter for different impulse durations, b) Pressure versus crack density $S_{v}$ for different impulse durations

One of the first fragmentation theories based on energy considerations were proposed by Grady [22] who considered the fragmentation of fluids. Glenn and Chudnovsky [18] extend this theory to solids. They notice that the governing 
mechanism for fragmentation is the release of the stored elastic energy. Grady [23,24] and Yarin [52] developed similar energy based fragmentation theories. In both theories, the fragment radius is expressed in terms of the strain rate and current density. The models in [22-24] are implemented by Johnson et al. [30] in the EPIC 2-code.

Recently, methods were developed which can handle dynamic failure by introducing discontinuities in the displacement field. In interelement separation models as proposed e.g. by $\mathrm{Xu}$ and Needleman [51], Camacho and Ortiz [7] and Ortiz et al. [42], cracks are introduced along existing interelement edges. Ortiz and coworkers have applied this class of methods successfully to dynamic fracture of brittle and ductile materials. One difficulty is that the crack paths will be aligned with the mesh orientation. Remeshing can overcome this difficulty but is computational expensive.

Another method which can handle complex failure is the extended finite element method, see e.g. Moes et al. [36,37]. The method was applied to dynamic problems in 2D by Belytschko et al. [5]. The method is ideally suited to study complex crack problems very efficiently and accurately. However, for problems with excessive cracking and fragmentation, the method will be computationally expensive.

Randles et al. [48] studied the fragmentation of cylindrical shells and bombs. They used a meshfree method and a plasticity model. Dynamic failure and fragmentation occurs quite naturally by separation of the particles. They were able to predict fragment sizes and fragment distributions of many experiments quite accurate. A problem is that the separation of particles can occur not only due to the material model but due to numerical artifacts. In [3], Belytschko et al. address the stability of meshfree methods and give improvements to significantly reduce the fragmentation due to numerical artifacts. However, they also point out that numerical instabilities may still arise if the deformations are too large, see also Rabczuk et al. [45].

\section{THE FRAGMENTATION MODEL}

We will follow an idea of Randles et al. [48] and simulate the failure of concrete with a meshfree method. Fragmentation occurs due to separation of the particles. The separation is governed by the constitutive model. In the following, we will describe the constitutive model for concrete and the meshfree method. 


\subsection{The Constitutive model for Concrete}

\subsubsection{General framework}

The constitutive law is a coupled damage-plasticity model. The rate-of-deformation tensor is decomposed into an elastic, a damage and a plastic part

$$
\dot{\boldsymbol{\epsilon}}=\dot{\boldsymbol{\epsilon}}_{e}+\dot{\boldsymbol{\epsilon}}_{d}+\dot{\boldsymbol{\epsilon}}_{p}=\dot{\boldsymbol{\epsilon}}_{e d}+\dot{\boldsymbol{\epsilon}}_{p}
$$

The rate equation for the stresses are given analogously by

$$
\boldsymbol{\sigma}_{e}^{\nabla}-\boldsymbol{\sigma}^{\nabla}=\mathbf{E}: \dot{\boldsymbol{\epsilon}}-\mathbf{E}: \dot{\boldsymbol{\epsilon}}_{e}=-\boldsymbol{\sigma}_{d}^{\nabla}-\boldsymbol{\sigma}_{p}^{\nabla}=\mathbf{E}: \dot{\boldsymbol{\epsilon}}_{d}+\mathbf{E}: \dot{\boldsymbol{\epsilon}}_{p}
$$

where the elastic and the damage strain rate is combined to $\dot{\boldsymbol{\epsilon}}_{e d}$ and $\boldsymbol{\sigma}^{\nabla}, \boldsymbol{\sigma}_{e}^{\nabla}$, $\boldsymbol{\sigma}_{d}^{\nabla}$ and $\boldsymbol{\sigma}_{p}^{\nabla}$ are frame indifferent stress rates.

The damage loading surface $F_{d}$ is formulated in the strain space and is decoupled from the plasticity loading surface $F_{p}$. The plastic flow rule does not determine the relaxation stress $\dot{\boldsymbol{\sigma}}_{p}$ but directly the plastic strain increment $\dot{\boldsymbol{\epsilon}}_{p}$

$$
\begin{aligned}
& \dot{\boldsymbol{\epsilon}}_{p}=\dot{\lambda}_{p} \mathbf{m}_{p}, \quad \dot{\boldsymbol{\sigma}}_{p}=-\dot{\lambda}_{p} \mathbf{E}: \mathbf{m}_{p} \\
& \dot{\boldsymbol{\sigma}}_{d}=\dot{\lambda}_{d} \mathbf{m}_{d}, \quad \dot{\boldsymbol{\epsilon}}_{d}=-\dot{\lambda}_{d} \mathbf{E}^{-1}: \mathbf{m}_{d}
\end{aligned}
$$

where $\dot{\lambda}_{p}$ and $\dot{\lambda}_{d}$ are the plastic and the damage multipliers which determine the size of the plastic strain and damage strain increment, respectively, and $\mathbf{m}_{p}$ and $\mathbf{m}_{d}$ give the direction of the plastic strain and damage strain increment, respectively. Loading is distinguished from unloading by the Kuhn-Tuckerconditions $F \leq 0, \dot{\lambda} \geq 0, F \dot{\lambda}=0$. From the differential consistency condition

$$
\begin{aligned}
& \dot{F}_{p}=\frac{\partial F_{p}}{\partial \boldsymbol{\epsilon}}: \dot{\boldsymbol{\epsilon}}+\frac{\partial F_{p}}{\partial \lambda_{p}} \dot{\lambda}_{p}=\mathbf{n}_{p}: \dot{\boldsymbol{\epsilon}}-H_{p} \dot{\lambda}_{p}=0 \\
& \dot{F}_{d}=\frac{\partial F_{d}}{\partial \boldsymbol{\epsilon}_{e}}: \dot{\boldsymbol{\epsilon}}_{e d}+\frac{\partial F_{d}}{\partial \lambda_{d}} \dot{\lambda}_{d}=\mathbf{n}_{d}: \dot{\boldsymbol{\epsilon}}_{e d}-H_{d} \dot{\lambda}_{d}=0
\end{aligned}
$$

the multipliers are gained and set in the flow rule, eq. (3) and (4)

$$
\begin{aligned}
& \dot{\boldsymbol{\epsilon}}_{p}=\frac{\mathbf{m}_{p} \otimes \mathbf{n}_{p}}{H_{p}}: \dot{\boldsymbol{\epsilon}}=-\mathbf{T}_{p}: \dot{\boldsymbol{\epsilon}} \\
& \dot{\boldsymbol{\sigma}}_{d}=\frac{\mathbf{m}_{d} \otimes \mathbf{n}_{d}}{H_{d}}: \dot{\boldsymbol{\epsilon}}_{e d}=\mathbf{T}_{d}: \dot{\boldsymbol{\epsilon}}_{e d}
\end{aligned}
$$


where $\mathbf{n}_{p}$ and $\mathbf{n}_{d}$ are the normals on the plastic and the damage surface, respectively. We assume an associated flow rule, i.e. $\mathbf{n}=\mathbf{m}$. Finally, a term for the tangential stiffness is obtained:

$$
\mathbf{E}_{t}=\left(\mathbf{E}+\mathbf{T}_{d}\right):\left(\mathbf{I}+\mathbf{T}_{p}\right)
$$

where $\mathbf{I}$ is the fourth order unity tensor and $\mathbf{T}_{p}$ and $\mathbf{T}_{d}$ are explained in eq. (7) and (8), respectively. The stresses are updated by

$$
\boldsymbol{\sigma}^{\nabla}=\mathbf{E}_{t}: \dot{\boldsymbol{\epsilon}}
$$

\subsubsection{Loading surfaces}

The constitutive relations are supplemented with evolution laws for damage and plasticity:

$$
F_{d}\left[\boldsymbol{\epsilon}, \kappa_{d}(\hat{D})\right]=\mathbf{D}, F_{p}\left[\boldsymbol{\epsilon}, \kappa_{p}\left(q_{p}\right)\right]=\boldsymbol{\epsilon}_{p}
$$

where $\kappa_{d}$ and $\kappa_{p}$ are the equivalent damage and plastic strain, respectively and $q_{p}$ and $\hat{D}$ are internal variables. We distinguish between isotropic damage in compression and anisotropic damage in tension. Hence, we will have a scalar damage variable $D$ for compression and an anisotropic damage tensor $\mathbf{D}$ in tension. The loading surfaces for damage are

$$
\begin{aligned}
& F_{d, c}=c_{1} J_{2}^{e}+\kappa_{d, c}\left(c_{2} \sqrt{J_{2}^{e}}+c_{3} \epsilon_{e, \max }^{(\alpha)}+c_{4} I_{1}^{e}\right)-\kappa_{d, c}^{2}=0 \\
& F_{d, t}=0.5 \boldsymbol{\epsilon}_{e}^{+}: \boldsymbol{\epsilon}_{e}^{+}-0.5 \kappa_{d, t}^{2}=0
\end{aligned}
$$

where the subscript $t$ and $c$ denote tension and compression, respectively, $c_{1}$ to $c_{4}$ are parameters of the damage surface, $I_{1}^{e}$ is the first invariant of the elastic strain tensor, $J_{2}^{e}$ is the second invariant of $\operatorname{dev} \boldsymbol{\epsilon}_{e}, \epsilon_{e, \max }^{(\alpha)}$ is the maximum $\alpha$ -th characteristic value of $\boldsymbol{\epsilon}_{e}$ and $\boldsymbol{\epsilon}_{e}^{+}$is the positive projection of the elastic strain tensor. Ortiz [41] decomposes the elasticity tensor in an uncracked part $\mathbf{E}_{0}$ and into a cracked part $\mathbf{E}^{+}$where $\mathbf{E}^{+}$describes the deformability of the microcracks. With an orthogonal projections $\mathbf{P}^{+}$of the strain space onto $\mathbf{E}^{+}$, the closest point in $\mathbf{E}^{+}$is associated to a given strain state, see figure 2 . This separates the negative components of the eigenvalues of $\boldsymbol{\epsilon}$. The projection is

$$
\epsilon_{e}^{+}=\mathbf{P}^{+}: \boldsymbol{\epsilon}_{e} \text { and } \boldsymbol{\epsilon}_{e}^{-}=\boldsymbol{\epsilon}_{e}-\boldsymbol{\epsilon}_{e}^{+}
$$

and

$$
\mathbf{P}^{+}=\sum_{\alpha} H\left(\epsilon^{(\alpha)}\right) \mathbf{d}^{(\alpha)} \otimes \mathbf{d}^{(\alpha)} \otimes \mathbf{d}^{(\alpha)} \otimes \mathbf{d}^{(\alpha)}
$$


The parameters $c_{1}$ to $c_{4}$ are calibrated at standard tests so that biaxial and triaxial failure surfaces according to curves from the literature (see e.g. Kupfer et al. [32] or Mills and Zimmermann [35]) can be reproduces. The evolution of the plastic part is given by

$$
F_{p}=\frac{1}{2}\left(\boldsymbol{\epsilon}_{e}^{+}: \boldsymbol{\epsilon}_{e}^{+}+c_{c} \boldsymbol{\epsilon}_{e}^{-}: \boldsymbol{\epsilon}_{e}^{-}\right)-\frac{1}{2} \kappa_{p}^{2}=0
$$

where $c_{c}$ is the cross-effect constant (see also Ortiz [41]).

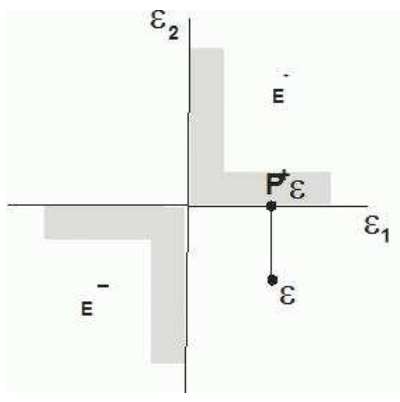

Fig. 2. Projection of the strain tensor

At this point, we would like to make some comments regarding the strain tensor used e.g. in the loading surfaces eqs. (11) to (13). We use Cauchy stresses in our computations and the rate-of-deformation tensor is the power conjugate tensor. Elasto-plastic material behavior for small elastic strains, large plastic strains and large rotations can be modelled with the logarithmic strain $\boldsymbol{\epsilon}=\ln \mathbf{V}$, where $\mathbf{V}$ is the left stretch tensor obtained from the polar decomposition of $\mathbf{F}=\mathbf{V} \cdot \mathbf{R}$. Since the assumption of small elastic strains in our model is applicable, we proceed as follows: The logarithmic strains can be approximated by integration of the rate-of-deformation tensor. The strains are rotated with the incremental rotation tensor $\Delta \mathbf{R}$ with the Hughes and Winget [29] algorithm:

$$
\boldsymbol{\epsilon}^{n+1}=\Delta \mathbf{R} \cdot \boldsymbol{\epsilon}^{n} \cdot \Delta \mathbf{R}+\Delta \boldsymbol{\epsilon}
$$

with

$$
\Delta \boldsymbol{\epsilon}=\ln \Delta \mathbf{V}, \quad \Delta \mathbf{V}=\mathbf{V}^{n}+1 \cdot\left(\mathbf{V}^{n}\right)^{-1}
$$

According to [29], $\Delta \mathbf{R}$ can be computed by

$$
\Delta \mathbf{R}=(\mathbf{1}-0.5 \Delta \mathbf{w}) \cdot(\mathbf{1}+0.5 \Delta \mathbf{w})
$$

with

$$
\Delta \mathbf{w}=0.5\left(\Delta \mathbf{u} \otimes \nabla^{n+1 / 2}-\nabla^{n+1 / 2} \otimes \Delta \mathbf{u}\right)
$$


Note that all tensorial variables in the constitutive model are rotated that way at the beginning of every increment.

\subsubsection{Evolution laws}

The damage evolution is described by a Weibull distribution:

$$
\hat{D}\left(\kappa_{d}\right)=1-e^{-\left(\frac{\kappa_{d}-e_{0}}{e_{d}}\right)^{g_{d}}} \quad \kappa_{d} \geq e_{0} \quad \text { and } \quad \hat{D}\left(\kappa_{d}\right)=0 \quad \kappa_{d}<e_{0}
$$

with $e_{0}, e_{d}$ and $g_{d}$ as material parameters. Isotropic compressive damage can also occur under tensile loading conditions and the evolution for compressive damage is given by

$$
\begin{aligned}
& D_{c}^{t+d t}=\max \left(\left(\hat{D}\left(\kappa_{d, c}\right), D_{c}^{t}\right) \quad(\text { compression })\right. \\
& D_{c}^{t+d t}=\max \left(\left(\hat{D}\left(\kappa_{d, c} / r_{c}\right), D_{c}^{t}\right) \quad(\text { tension })\right.
\end{aligned}
$$

More details are given in [43] and [44].

The evolution of the anisotropic (tensile) damage tensor $\mathbf{D}_{t}$ is defined in the direction of the positive principal strain. The damage state is stored in form of a complementary continuity tensor a. Hence, the damage tensor in the direction of the principal strain $\alpha$ is

$$
D_{t}^{t,(\alpha)}=1-\mathbf{d}^{(\alpha)} \cdot \mathbf{a} \cdot \mathbf{a} \cdot \mathbf{d}^{(\alpha)}
$$

New tensile damage occurs in the direction of the positive principal strain, weighted with a factor $r_{e}$ to take into account the direction where the maximum damage increases:

$$
D_{t}^{t+d t,(\alpha)}=\max \left(\hat{D}\left(\kappa_{d, t} \cdot r_{e}\right), D_{t}^{t,(\alpha)}\right) \quad(\text { tension })
$$

with

$$
r_{e}=\frac{<\boldsymbol{\epsilon}_{e}^{(\alpha)}>}{\max \boldsymbol{\epsilon}_{e}^{(\alpha)}}
$$

where $<>$ are the McAuly brackets and the subscript $t$ indicates tension. According to eq. (23), tensile damage can occur under compressive loading as well:

$$
D_{t}^{t+d t,(\alpha)}=\max \left(\hat{D}\left(\max \left[<\boldsymbol{\epsilon}^{(\alpha)}>/ r_{t}, \kappa_{d, t} / r_{t}\right]\right), D_{t}^{t,(\alpha)}\right)
$$


The reduction factor $r_{t}$ is far smaller than $r_{c}$. The deformation rate for the tensile damage according to eq. (27) is:

$$
\dot{\mathbf{D}}_{t}=\sum_{\alpha} \dot{D}_{t}^{(\alpha)} \mathbf{d}^{(\alpha)} \otimes \mathbf{d}^{(\alpha)}
$$

The principal direction of the rate of the tensile damage coincides with the one of the elastic strain tensor, but it is not necessarily the case for the total tensile damage tensor, since it depends on the complete loading history. The hardening modulus $H_{d}$ is

$$
H_{d, t}=\frac{\partial F_{d, t}}{\partial \lambda_{d}}=\frac{\partial F_{d, t}}{\partial D_{t, \max }^{(\alpha)}}=\frac{\partial F_{d, t}}{\partial \kappa_{d, t}} \cdot \frac{\partial \kappa_{d, t}}{\partial D_{t, \max }^{(\alpha)}}
$$

From eq. (28), the continuity rate can be computed by

$$
\dot{\mathbf{a}}=\sum_{\alpha} \sqrt{\left(1-\dot{D}_{t}^{(\alpha)}\right)} \mathbf{d}^{(\alpha)} \otimes \mathbf{d}^{(\alpha)}
$$

The calculation of the integral of eq. (30) to obtain the total continuity tensor is not permitted, since under certain circumstances continuities $<0$, that means damages $>1$ can occur. Therefore, the continuity tensor $\mathbf{a}$ is computed by

$$
\mathbf{a}=\prod_{t} d \mathbf{R}_{\mathbf{a}}
$$

with

$$
d \mathbf{R}_{\mathbf{a}}=\sum_{\alpha} \frac{a^{t+d t,(\alpha)}}{a^{t,(\alpha)}} \mathbf{d}^{(\alpha)} \otimes \mathbf{d}^{(\alpha)}=\sum_{\alpha} \sqrt{\frac{1-D_{t}^{t+d t,(\alpha)}}{1-D_{t}^{t,(\alpha)}}} \mathbf{d}^{(\alpha)} \otimes \mathbf{d}^{(\alpha)}
$$

Eq. (32) guarantees, that every damage increment in direction of the current principal strain reduces the continuity, but simultaneously the principal values of a cannot be negative. The relation between the internal plastic variable $q_{p}$ and the plastic equivalent strain $\kappa_{p}$ is described in eq. (33):

$$
q_{p}\left(\kappa_{p}\right)=c_{p}\left(\kappa_{p}-e_{d}\right)\left(1-e^{-\left(\frac{\kappa_{p}-e_{d}}{e_{p 1}}\right)}\right) e^{-\frac{\kappa_{p}}{e_{p 2}}} \forall \kappa_{p} \geq e_{d}
$$

with material constants $e_{d}, e_{p 1}, e_{p 2}$ and $c_{p}$. The parameter $c_{p}$ determines the relation of the internal plastic variable $q_{p}$ to the equivalent plastic strain $\kappa_{p}$ and must be chosen $<1$. Otherwise, the plastic strain tensor maybe larger than 
the total strain tensor. The parameter $e_{p 1}$ determines the accumulation and $e_{p 2}$ determines the decay of the internal plastic variable at large deformations. The hardening modulus $H_{p}$ is

$$
H_{p}=-\frac{\partial F_{p}}{\partial \lambda_{p}}=-\frac{\partial F_{p}}{\partial \kappa_{p}} \cdot \frac{\partial \kappa_{p}}{\partial q_{p}} \cdot \frac{\partial q_{p}}{\partial \lambda_{p}}=\kappa_{p} \cdot \frac{\partial \kappa_{p}}{\partial q_{p}} \cdot \sqrt{\left(\boldsymbol{\epsilon}_{e}^{+}+c_{c} \boldsymbol{\epsilon}_{e}^{-}\right):\left(\boldsymbol{\epsilon}_{e}^{+}+c_{c} \boldsymbol{\epsilon}_{e}^{-}\right)}(
$$

Now, the normals onto the loading surfaces can be computed which are necessary to obtain the tangential stiffness. The normal $\mathbf{n}_{d}$ of the damage surface is obtained by taking of derivatives $F_{d}$ with respect to the elastic strain tensor $\boldsymbol{\epsilon}_{e}$ as

$$
\begin{aligned}
& \mathbf{n}_{d, c}=\left(c_{1}+\frac{\kappa_{d, c} c_{2}}{2 \sqrt{J_{2}}}\right) \operatorname{dev} \boldsymbol{\epsilon}_{e}+\kappa_{d, c}\left(c_{3} \mathbf{d}^{(\alpha)} \otimes \mathbf{d}^{(\alpha)}+c_{4} \mathbf{I}\right) \\
& \mathbf{n}_{d, t}=\boldsymbol{\epsilon}_{e}^{+}
\end{aligned}
$$

The normal of the plastic surface is obtained by taking derivatives of $F_{p}$ with respect to the elastic strain tensor:

$$
\mathbf{n}_{p}=\frac{\partial F_{p}}{\partial \boldsymbol{\epsilon}}=\frac{\partial F_{p}}{\partial \boldsymbol{\epsilon}_{e}^{+}}: \frac{\partial \boldsymbol{\epsilon}_{e}^{+}}{\partial \boldsymbol{\epsilon}}+\frac{\partial F_{p}}{\partial \boldsymbol{\epsilon}_{e}^{-}}: \frac{\partial \boldsymbol{\epsilon}_{e}^{-}}{\partial \boldsymbol{\epsilon}}=\boldsymbol{\epsilon}_{e}^{+}+c_{c} \boldsymbol{\epsilon}_{e}^{-}
$$

To account for a decayed crack propagation which we attribute to inertia effects, a dynamic damage $\hat{D}_{d y n}$ is introduced which decays the static damage evolution:

$$
\hat{D}_{d y n}=\int_{\tau=0}^{t} \frac{\partial \hat{D}}{\partial \tau} H(t-\tau) d \tau
$$

The history function $H(t-\tau)$ governing the activation of newly developed damage can be derived from rheological models taking into account the mechanics of microcrack opening. Figure 3 shows a double mass model with a non-linear crack-opening damper for the viscous moisture effect, a mass damper for the kinetic energy dissipation and a spring for the elastic stiffness. The two masses represent the undamaged elastic zones adjacent to the micro-defect. The solution of the differential equation leads to a history function based on this internal physical mechanism. As the limited amount of dynamic load-history data does not justify the rather complicated parameter identification in this sophisticated model and as the computational effort is quite considerable, simpler decay function should be used for $H$. An exponential Maxwell relaxation is chosen, where the relaxation time $\tau$ depends on the equivalent strain rate 


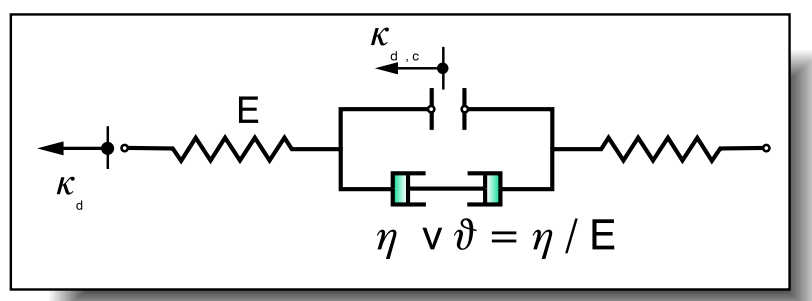

Fig. 3. Rheological model: $\vartheta$ is the relaxation time, $v$ the crack velocity, $\eta$ the viscosity

at time $\vartheta$ when new microdamage developes. The history function can be formulated as follows:

$$
H(t-\tau)=e^{-\left(\frac{t-\tau}{\vartheta\left(\kappa_{d}^{*}\right)}\right)^{g_{h}}}
$$

with $g_{h}$ as material parameter. The history function $H$ decays monotonously from 1 to 0 . The relaxation time is defined by an empirical function of the equivalent elastic strain rate $\dot{\kappa}_{d}^{*}$ related to a reference value $\dot{\kappa}_{0}$

$$
\vartheta\left(\dot{\kappa}_{d}^{*}\right)=\vartheta_{0} \cdot \dot{\kappa}_{d}^{* c_{t 1} l n} \dot{\kappa}_{d}^{*}-c_{t 2} \quad \text { and } \quad \dot{\kappa}_{d}^{*}=\frac{\dot{\kappa}_{d}(\tau)}{\dot{\kappa}_{0}} \quad \text { with } \quad \dot{\kappa}_{0}=1 s^{-1}
$$

with $c_{t 1}$ and $c_{t 2}$ as material parameters. With this Maxwell relaxation the dynamic strength increase can be approximated in a wide range of strain rates. Note that the introduction of a viscosity also regularizes the equations and removes mesh dependence.

\subsubsection{Concrete Hugoniot}

Under high hydrostatic pressure, the damaged concrete behaves like a granular material. This is taken into account by modifying the elastic modulus with a stiffening function $\gamma$ :

$$
\begin{aligned}
& \gamma=\left(1-a_{v}\left(1-e^{-\beta}\right)\right)\left(1+\left(\frac{\beta}{b_{v}}\right)^{2}\right) \forall \operatorname{tr} \boldsymbol{\epsilon}_{e}<-e_{v, t h} \\
& \gamma=1 \quad \forall \operatorname{tr} \boldsymbol{\epsilon}_{e} \geq-e_{v, t h}
\end{aligned}
$$

with

$$
\beta=\frac{\left|\operatorname{tr} \boldsymbol{\epsilon}_{e}\right|-e_{v, t h}}{e_{v}}
$$


and $e_{v}, e_{v, t h}, b_{v}$ and $a_{v}$ as material parameters. The material parameters are calibrated so that the data obtained from the Hugoniot-tests performed by Ockert [40] are reproduced.

\subsection{The MLSPH-Method}

The MLSPH method is an improvement of the SPH method $([34,17])$ as proposed by Dilts [13]. It is similar to the element-free Galerkin method with nodal integration, see Belytschko et al. [4]. An MLS approximation is given by

$$
u^{h}(\mathbf{x})=\sum_{J \in S} \mathbf{p}_{J}(\mathbf{x}) \mathbf{a}(\mathbf{x})
$$

where $S$ is a set of supporting nodes, $\mathbf{p}$ is the basis vector and $\mathbf{a}$ are parameters chosen to minimize

$$
J=\sum_{J \in S}\left(\mathbf{p}_{J}(\mathbf{x})^{T} \mathbf{a}(\mathbf{x})-u_{J}\right)^{2} W\left(\mathbf{x}-\mathbf{x}_{J}, h\right) .
$$

where $W$ is the kernel function. Minimizing equation (45) with respect to a leads to the approximation

$$
u^{h}(\mathbf{x})=\sum_{J \in S} u_{J} \Phi_{J}(\mathbf{x})
$$

with

$$
\begin{aligned}
& \Phi_{J}=\mathbf{p}(\mathbf{x})^{T} \cdot \mathbf{A}(\mathbf{x})^{-1} \cdot \mathbf{p}_{J}(\mathbf{x}) W\left(\mathbf{x}-\mathbf{x}_{J}, h\right) \\
& \mathbf{A}(\mathbf{x})=\sum_{J \in S} \mathbf{p}_{J}(\mathbf{x}) \mathbf{p}_{J}^{T}(\mathbf{x}) W\left(\mathbf{x}-\mathbf{x}_{J}, h\right)
\end{aligned}
$$

The basis vector $\mathbf{p}$ contains the functions which are reproduced. Usually, constant or linear functions are chosen since they guarantee the global conservation of linear momentum (for constant functions) and angular momentum (for linear functions). We have chosen linear functions:

$$
\mathbf{p}(\mathbf{x})=\left(\begin{array}{llll}
1 & x & y & z
\end{array}\right) \quad \forall \mathbf{x} \in \Re^{3} .
$$

The shape functions $\Phi$ are used for the discretization of the test functions $\delta \mathbf{v}$ and trial functions $\mathbf{v}$ in the Galerkin weak form. Crucial is the linear 
momentum equation which is given by

$$
-\int_{\Omega}(\nabla \delta \mathbf{v})^{T}: \boldsymbol{\sigma} d \Omega+\int_{\Omega} \delta \mathbf{v} \cdot(\varrho \mathbf{b}-\varrho \dot{\mathbf{v}}) d \Omega+\int_{\Gamma_{t}} \delta \mathbf{v} \cdot \overline{\boldsymbol{\tau}} d \Gamma=0 .
$$

where $\boldsymbol{\sigma}$ is the Cauchy stress tensor, $\mathbf{b}$ are the body forces, $\dot{\mathbf{v}}$ is the acceleration, $\varrho$ is the current density and $\overline{\boldsymbol{\tau}}$ are the applied tractions. The shape functions in this section are expressed in terms of spatial coordinates. Belytschko et al. [3] have shown that this can cause instabilities and propose to express all variables in terms of material coordinates. This guarantees that fracture happens due to the material model and not due to numerical artifacts. However, for large deformations, instabilities occur since the domain of influence is extremely distorted in the current configuration.

In many particle methods, the discrete equations are obtained by use of a nodal integration. This is another source for numerical instabilities (due to rank deficiency). Belytschko et al. [3] have shown that adding stress points to the particles can remove this instability.

\section{RESULTS}

\subsection{Concrete under impact loading}

Hanchak et al. [25] performed different impact experiments of reinforced concrete slabs. In their experiments, the concrete had a compressive strength of $48 M P A$ and $140 M P A$, respectively. They used a $0.53 \mathrm{~kg}$ steel projectile which was shot onto a $0.178 m$ thick $0.61 m \times 0.61 m$ concrete slab, see figure 4 . Besides the initial velocity of the steel projectile, they also measured its discharge velocity. The influence of the reinforcement $(5.7 \mathrm{~mm}$ diameter, $\mathrm{e}=76$ $\mathrm{mm}$ ) with respect to the perforation resistance can be neglected according to Hanchak et al. [25]. Interesting is the observation, that the concrete with a strength of $140 \mathrm{MPa}$ was able to increase the perforation resistance only slightly with respect to the concrete with a compressive strength of $48 \mathrm{MPa}$. The experimental setup is shown in figure 4.

The reinforcement is modelled with an elasto-plastic material law with a strain based failure. Simplified, a rigid bond between concrete and the reinforcement is assumed. The concrete is modelled with the material model from section 3.1. We consider different impact velocities as shown in table 2 and focus on the concrete slabs with a compressive strength of $48 M P a$. Similar results were obtained for the other slabs. The material parameters can be found in 


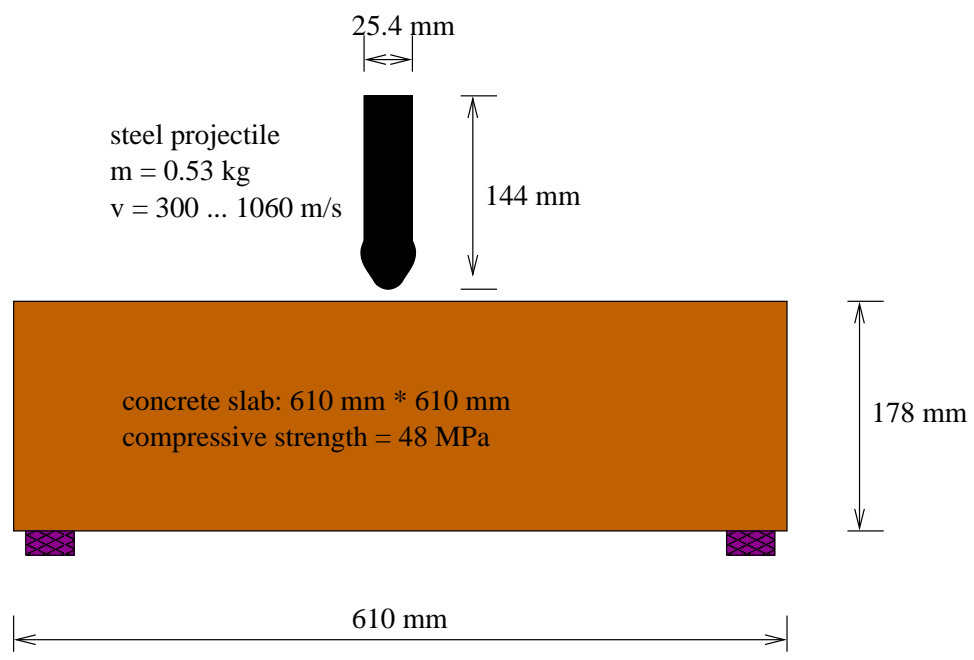

Fig. 4. Experimental setup of the impact experiments preformed by Hanchak et al. $[25]$

Table 1

Material parameters of the constitutive model for concrete

\begin{tabular}{|c|c||c|}
\hline$E_{0}=36,000 \mathrm{MPa}$ & $e_{0}=2.5 \cdot 10^{-5}$ & $e_{p 2}=0.4$ \\
\hline$\nu=0.22$ & $e_{d}=2.2 \cdot 10^{-4}$ & $\varrho=2.4 \cdot 10^{-3}$ \\
\hline$c_{1}=0.0123424$ & $g_{d}=1.5$ & $n_{d a m}=70$ \\
\hline$c_{2}=0.025166$ & $r_{t}=1.2$ & $a_{v}=0.7$ \\
\hline$c_{3}=0.782058$ & $r_{c}=20$ & $b_{v}=3.5$ \\
\hline$c_{4}=0.346384$ & $c_{p}=0.9$ & $e_{v}=0.02$ \\
\hline$c_{c}=0.08$ & $e_{p 1}=1.1 \cdot 10^{-3}$ & $e_{v, t h}=0.008$ \\
\hline
\end{tabular}

Table 2

The different impactor velocities of the Hanchak plates and the corresponding discretizations

\begin{tabular}{|l||c||c|}
\hline & number of particles & initial impact velocity $[\mathrm{m} / \mathrm{s}]$ \\
\hline \hline H1 (2D) & 27,540 & 360 \\
\hline H2 (2D) & 27,540 & 430 \\
\hline H3 (2D) & 27,540 & 750 \\
\hline H4 (2D) & 27,540 & 1060 \\
\hline H5 (2D) & 112,900 & 750 \\
\hline H6 (3D) & 296,280 & 1060 \\
\hline \hline
\end{tabular}

table 1. The impactor and the concrete slab is simplified discretized in $2 \mathrm{D}$ under the assumption of plane strain conditions but also 3D computations were performed, see table 2 . 
Figure 5 shows the deformed concrete plate $\mathrm{H} 4$ at different time steps. It can be seen that the concrete slab is completely perforated which matches well with the experiment.

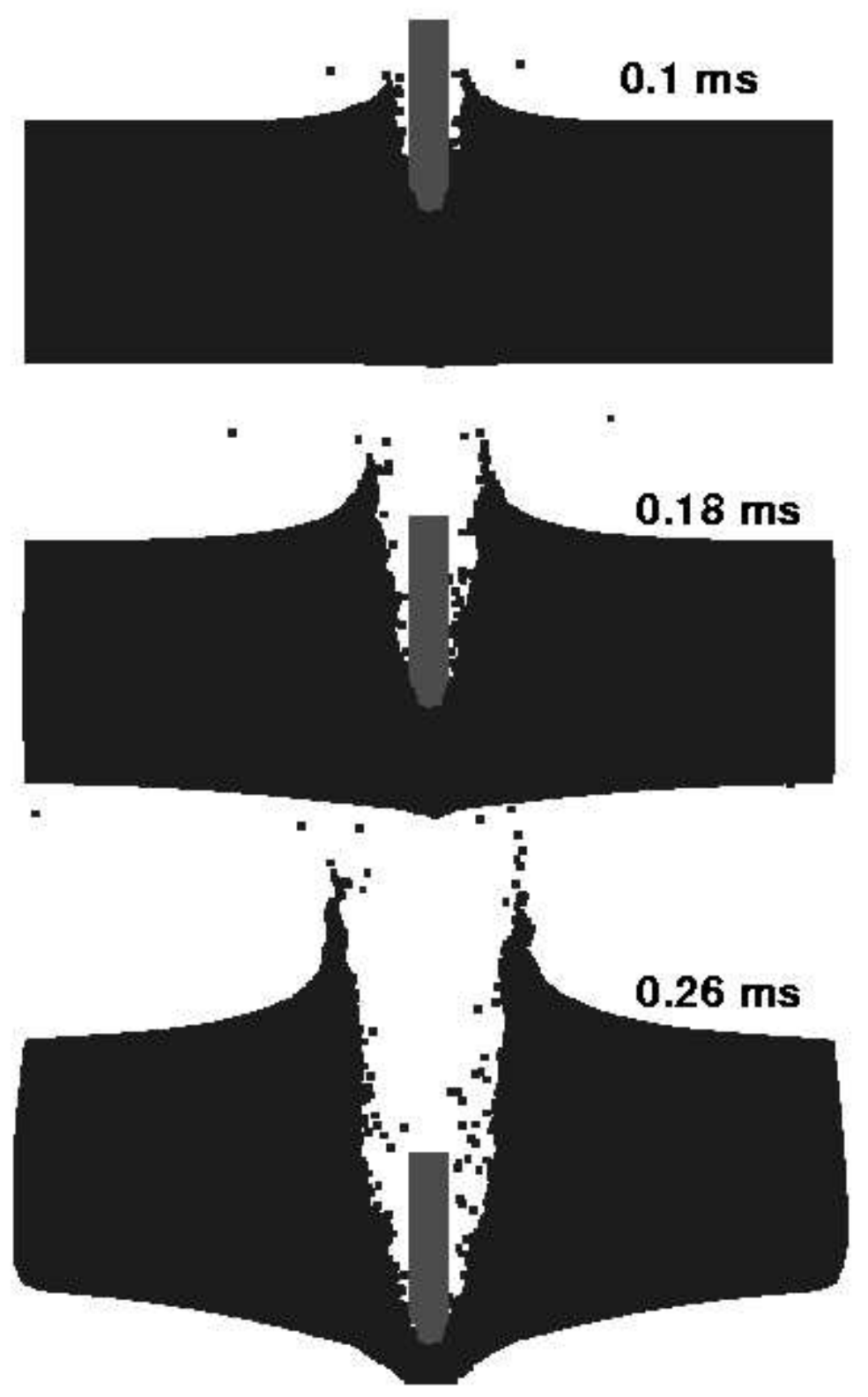

Fig. 5. Deformed configuration of the Hanchak slab $\mathrm{H} 4$ a) at $0.1 \mathrm{~ms}$, b) at $0.18 \mathrm{~ms}$, c) at $0.26 \mathrm{~ms}$

Figure 6 compares the ejection velocity of the Hanchak experiments with the simulations. It shows the velocity profile of the impactor in dependence of the penetration depth for different impact velocities. It can be seen, that the numerical simulation was able to reproduce the measured ejection velocity very well. Two computation with different number of particles for an impact 
velocity of $750 \mathrm{~m} / \mathrm{s}$ (H3 and $\mathrm{H} 5$, see table 2) were carried out. The results are reproduced quite mesh independent.

The two dimensional calculation with 27,540 particles (H4) is compared with the three dimensional calculation (H6). Because of the higher resolution of the slab, the two dimensional simulation is able to reproduce the experimental data better than the three dimensional simulation. For the two-dimensional simulation $\mathrm{H} 4$, the distance between the particles is $2.0 \mathrm{~mm}$ while for the three dimensional simulation (H6) the distance between the particles is $6.0 \mathrm{~mm}$. For the same resolution in 3D (compared to simulation $\mathrm{H} 4$ ), more than 8.4 million particles would be necessary. We also carried out a $2 \mathrm{D}$ simulation with a particle separation of $6.0 \mathrm{~mm}$ which gave a discharge velocity of approximately $810 \mathrm{~m} / \mathrm{s}$ which is slightly below the discharge velocity of the 3D simulation. However, at a certain refinement of the plate, convergence is obtained as shown in the 2D case for an impact velocity of $750 \mathrm{~m} / \mathrm{s}$.

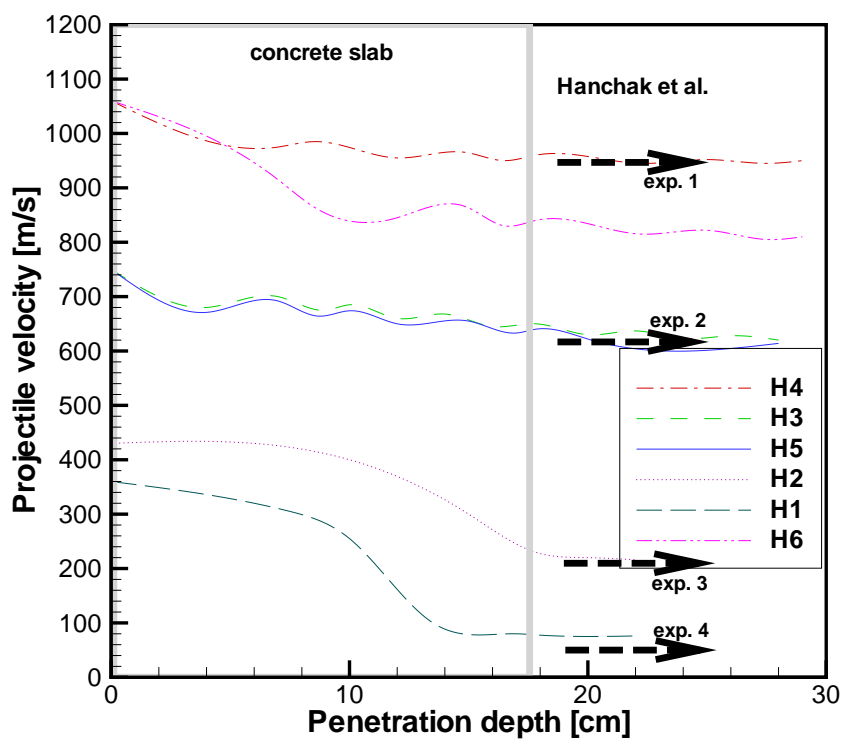

Fig. 6. Projectile velocity from the simulations compared to the ejection velocity measured by Hanchak et al. [25]

\subsection{Concrete under explosive loading}

At the Institute of Concrete Structure a series of explosion tests of concrete slabs with different thicknesses and strengths were performed by Ockert [40] and Hermann [28]. The numerical results are compared with data from two experiments (slab $E 1$ and $E 2$ ). The slabs were loaded with a plane wave generator which consists of an inner cone of TNT and an outer cone of Composition B. At different positions in the concrete slab hydrostatic pressure 
gauges were positioned. The measured pressures are compared with those obtained by the simulations. A schematic test setup is illustrated in figure $7 \mathrm{a}$, a typical discretization of the slab is shown in figure $7 \mathrm{~b}$. The yellow cone shows the explosive and the concrete slab is illustrated in blue. Approximately 320,000 particles for the concrete were used for slab $E 1$. For slab $E 2$, we used a coarse particle arrangement with 265,000 concrete particles and a fine particle arrangement with 740,000 concrete particles. A more detailed investigation about the convergence of the method in dependence of the particle number and the size of domain of influence is omitted here and can be found in Rabczuk $[47]$.

The concrete slab $E 1$ had a thickness of $30 \mathrm{~cm}$ and an area of $100 \mathrm{~cm} \times 100 \mathrm{~cm}$. The compressive strength of the concrete was $200 \mathrm{MPa}$. The parameters for the constitutive law are listed in table 3. The slab $E 2$ had a thickness of $32 \mathrm{~cm}$ and an area of $120 \mathrm{~cm} \times 120 \mathrm{~cm}$ but the compressive strength was only $48 \mathrm{MPa}$. The material parameters for this slab is the same as for the impact problem in the previous section and shown in table 1. Both concrete slabs were loaded with an explosive cone as shown in figure 7 . The cone has a diameter of $10.3 \mathrm{~cm}$ and its height is $7.5 \mathrm{~cm}$. The explosive is simulated via a John-Wilkonson-Lee (JWL) equation of state (EOS):

$$
p=A\left(1-\frac{\omega \varrho}{R_{1} \varrho_{0}}\right)^{-R_{1} \frac{\varrho_{0}}{\varrho}}+B\left(1-\frac{\omega \varrho}{R_{2} \varrho_{0}}\right)^{-R_{2} \frac{\varrho_{0}}{\varrho}}+\frac{\omega \varrho^{2}}{\varrho_{0}} E_{m 0}
$$

The material parameters for the explosive are given in table 4 .

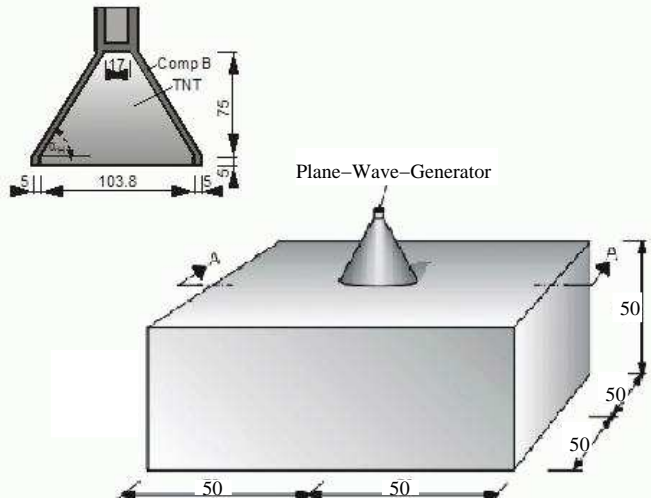

a)

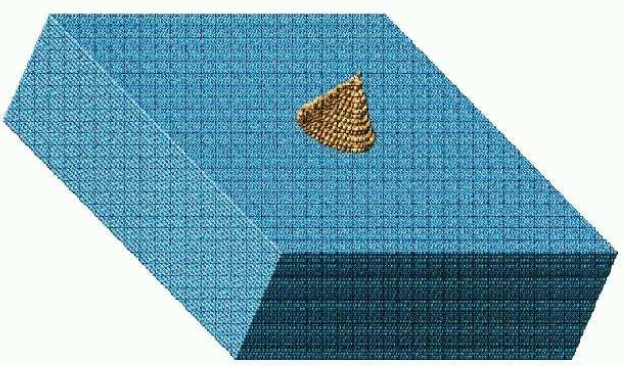

b)

Fig. 7. a) Schematic test set up of the explosive experiments, b) Discretization of the concrete slab

In figure 8a, the maximum pressures from the experiment $E 1$ (for different positions of the gauges) are compared with the pressures obtained from the numerical simulations. The simulation is able to predict the measured pressure quite well. For slab E2, we have only information about the damage of the 
Table 3

Material parameters of the constitutive model for concrete

\begin{tabular}{|c|c||c|}
\hline$E_{0}=73,000 \mathrm{MPa}$ & $e_{0}=1.0 \cdot 10^{-4}$ & $e_{p 2}=0.4$ \\
\hline$\nu=0.22$ & $e_{d}=2.9 \cdot 10^{-4}$ & $\varrho=2.8 \cdot 10^{-3}$ \\
\hline$c_{1}=0.0123424$ & $g_{d}=2.5$ & $n_{d a m}=70$ \\
\hline$c_{2}=0.025166$ & $r_{t}=1.2$ & $a_{v}=0.7$ \\
\hline$c_{3}=0.782058$ & $r_{c}=20$ & $b_{v}=3.5$ \\
\hline$c_{4}=0.346384$ & $c_{p}=0.9$ & $e_{v}=0.02$ \\
\hline$c_{c}=0.08$ & $e_{p 1}=1.1 \cdot 10^{-3}$ & $e_{v, t h}=0.008$ \\
\hline
\end{tabular}

Table 4

Material parameters of the explosive

\begin{tabular}{|c||c|c|c|c|c|c|c|c|c|c|}
\hline & $\varrho_{0}$ & $P_{\max }$ & $c_{D}$ & $E_{m 0}$ & $A$ & $B$ & $C$ & $R_{1}$ & $R_{2}$ & $\omega$ \\
\hline & {$\left[g / m m^{3}\right]$} & {$[M P a]$} & {$[m / s]$} & {$[M P a]$} & {$[M P a]$} & {$[M P a]$} & {$[M P a]$} & & & \\
\hline TNT & 0.00163 & 21,000 & 6930 & 70,000 & 37,120 & 3231 & 1045 & 4.15 & 0.95 & 0.3 \\
\hline Comp. B & 0.001717 & 29,500 & 7980 & 85,000 & 52,420 & 7678 & 1082 & 4.2 & 1.1 & 0.34 \\
\hline
\end{tabular}

slab.

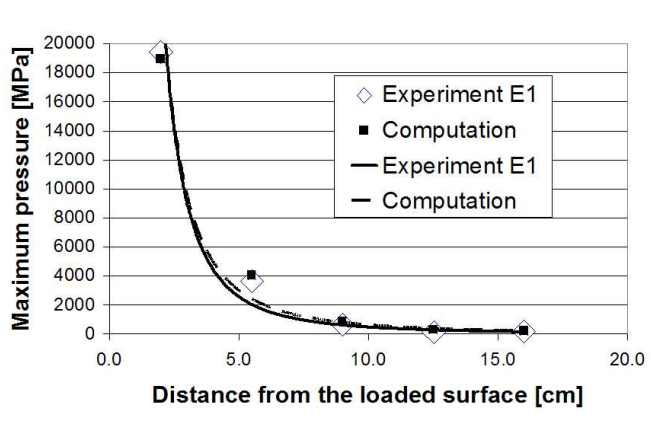

a)

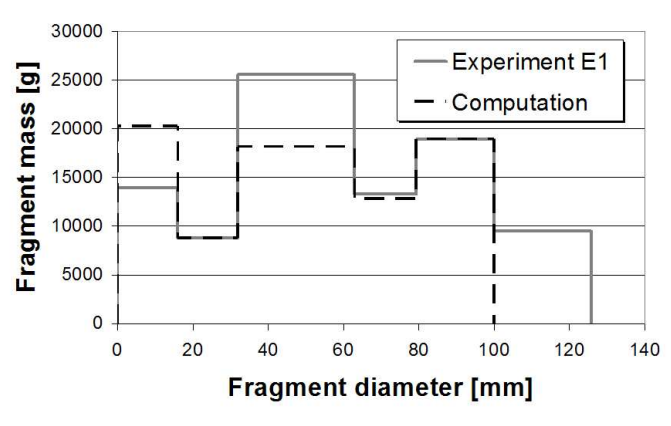

b)

Fig. 8. a) Maximum pressure of the gauges of the experiment $E 1$ and the numerical simulation, b) Fragment distribution of slab E1

In figures 9 and 11, the fragmentation process of the slabs in the simulations is shown. A more ductile fracture of slab $E 1$ can be seen compared to the fracture of $E 2$ where large cracks evolved. This behavior was also observed in the experiments, see figures 10 and 12 . Slab $E 2$ is completely perforated while a large damage of slab $E 1$ can be seen after the experiment. The simulation predicts a perforation of both slabs. This is a good approximation since slab $E 1$ perforated when we tried to lift it for transportation purposes. In table 5, the results from the computations and experiments are summarized. As can be seen, the results of the simulation of slab $E 2$ with different particle numbers give similar results. 


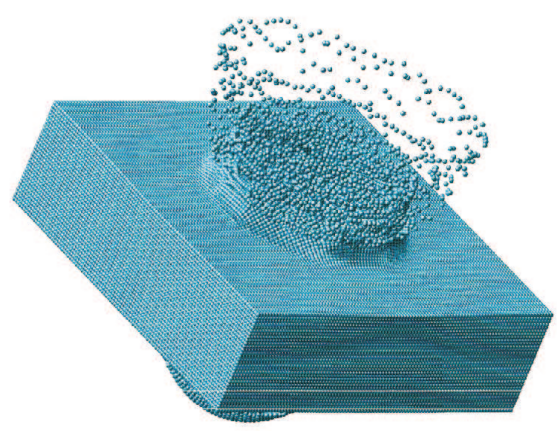

a)

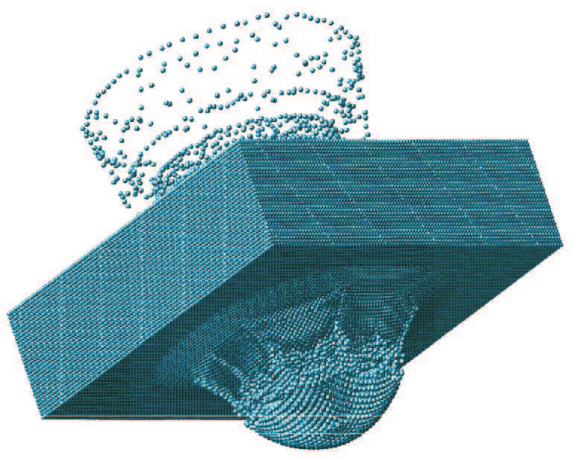

b)

Fig. 9. Fragmentation of the slab $E 1$ a) top view, b) bottom view

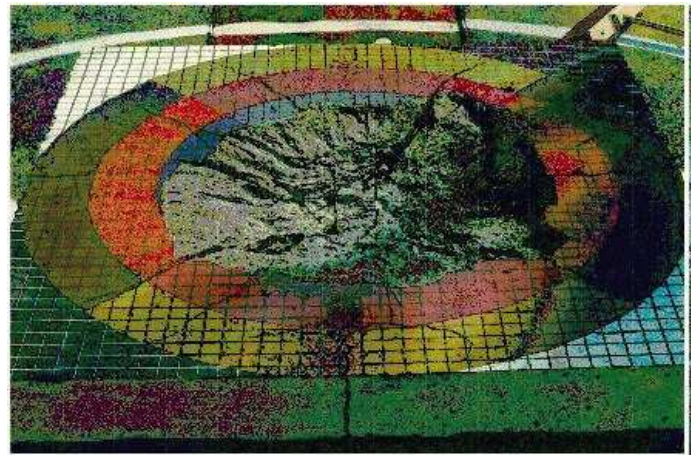

a)

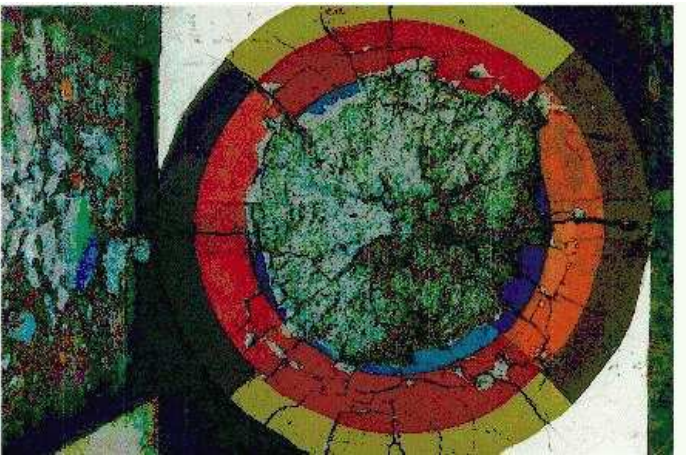

b)

Fig. 10. Damage of the slab $E 1$ after the experiment a) top view, b) bottom view

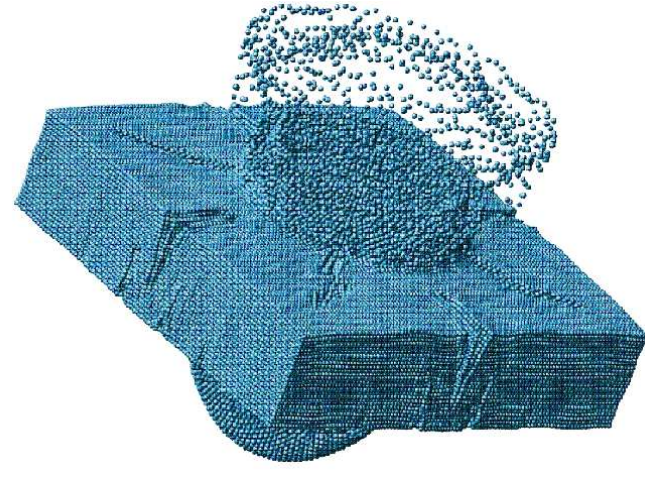

a)

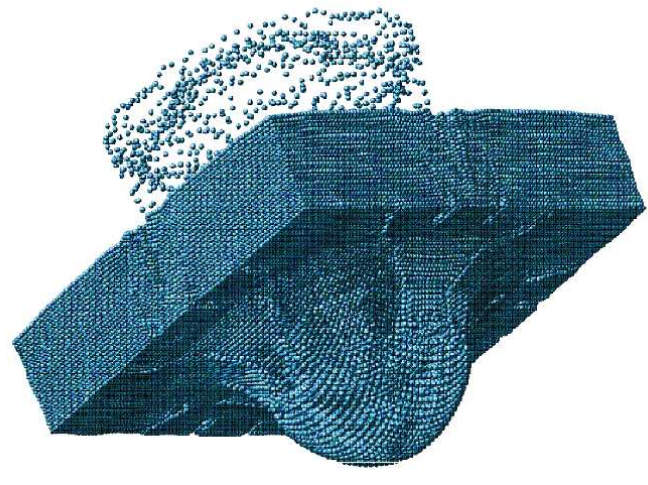

Fig. 11. Fragmentation of the slab $E 2$ (coarse particle arrangement) a) top view, b) bottom view

Finally the fragment distribution is of interest. Table 5 shows the total fragment mass for $E 1$. Figure $8 \mathrm{~b}$ gives the fragment distribution. The computation is able to predict the fragment distribution of the experiment quite well. At this point, it should be remarked that the experiments showed a large scatter, so only qualitative predictions can be made. It can be seen that the computation is able to predict the largest fragment size pretty well. 

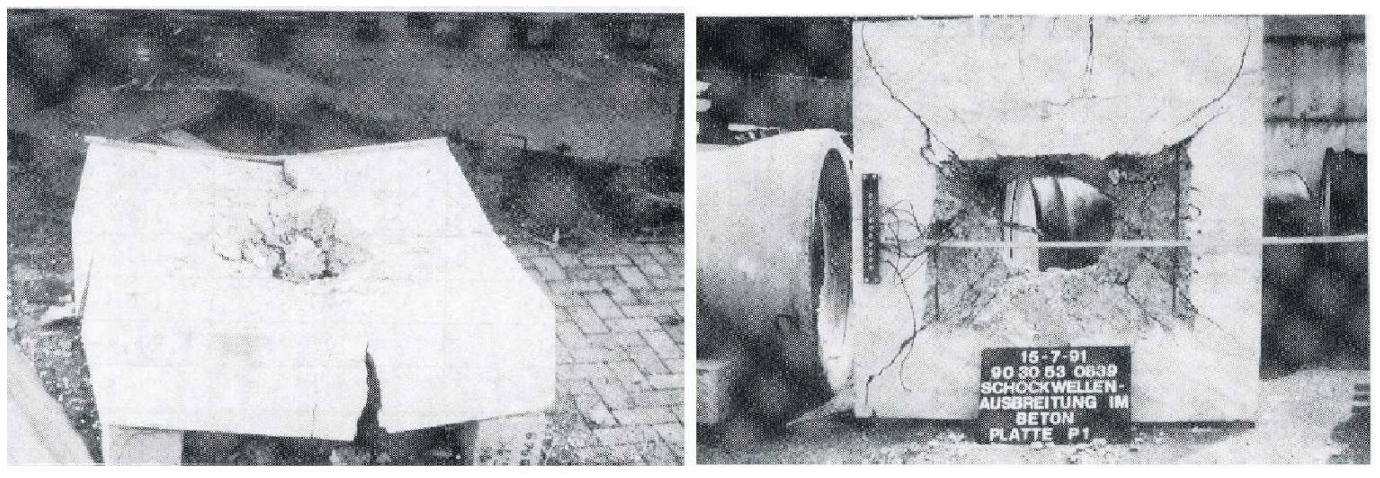

Fig. 12. Damage of the slab $E 2$ after the experiment a) top view, b) bottom view

Table 5

Summery of the results of the different SPH/MLSPH-simulations and the experiments

\begin{tabular}{|c|c||c||c||c||c|}
\hline & $\begin{array}{c}\text { Upper crater } \\
\text { diameter }\end{array}$ & $\begin{array}{c}\text { Upper crater } \\
\text { depth }\end{array}$ & $\begin{array}{c}\text { Lower crater } \\
\text { diameter }\end{array}$ & $\begin{array}{c}\text { Lower crater } \\
\text { depth }\end{array}$ & $\begin{array}{c}\text { Fragment } \\
\text { mass [kg] }\end{array}$ \\
\hline Exp. E1 & $50 \mathrm{~cm}$ & $7 \mathrm{~cm}$ & $55 \mathrm{~cm}$ & $17 \mathrm{~cm}$ & 90 \\
\hline Exp. E2 & $51 \mathrm{~cm}$ & perforation & $62 \mathrm{~cm}$ & perforation & NA \\
\hline Comp. E1 & $65 \mathrm{~cm}$ & perforation & $77 \mathrm{~cm}$ & perforation & 90 \\
\hline Comp. E2 (coarse) & $55 \mathrm{~cm}$ & perforation & $77 \mathrm{~cm}$ & perforation & NA \\
\hline Comp. E2 (fine) & $50 \mathrm{~cm}$ & perforation & $75 \mathrm{~cm}$ & perforation & NA \\
\hline
\end{tabular}

\section{SUMMARY}

In this paper we studied the dynamic failure of concrete structures. Therefore, a viscous damage model is proposed which can capture well the so-called strain rate effect for high dynamic loading conditions. It also regularizes the PDE and removes mesh dependence. One important feature of the viscous damage model is that the length scale introduced by the viscosity has a physical meaning. The viscous parameters of the model are calibrated so that experimental tensile and compressive strength increases are reproduced well.

A meshfree method is employed. Meshfree methods have several advantages over finite elements for problems such as dynamic fracture and fragmentation. First, they can easily handle large deformations because of the lack of a mesh and second, fragmentation happens naturally by separation of the particles according to the constitutive model.

The method is verified at impact events with different impactor velocity and explosion tests of concrete slabs. The experimental failure patterns are in good agreement with our numerical results. For the impact problems, we have 
obtained an excellent agreement between the experimental discharge velocity of the impactor and the discharge velocity of the impactor from our numerical simulation. Due to computational cost, we mainly performed 2D computations with the assumption of plane strain conditions. For one example, convergence in the discharge velocity of the impactor was shown. For the explosion tests, we compared experimental and computational results for pressure gauges which were located in the concrete and fragment distributions. We were able to capture the experimental pressures. The fragment distribution was reproduced qualitatively well. However, to model fragmentation in 3D is still a challenging task. Another method of treating the dynamic failure of brittle materials with meshfree methods and cohesive surfaces is proposed in 2D by Rabczuk and Belytschko [46]. A three dimensional (adaptive) version which also deals with fragmentation and impact problems is on the way.

\section{References}

[1] Aimone C.T., Meyers M.A.: Rock mechanics in Productivity design, eds. Dowding D.H., Singh M.M., AIME, Warrendale, PA, 1982, 979

[2] Bachmann H.: Die Massenträgheit in einem Pseudo-Stoffgesetz für Beton bei schneller Zugbeanspruchung, Institut für Massivbau und Baustofftechnologie, Universität Karlsruhe, 1993

[3] Belytschko T., Guo Y., Liu W.K., Xiao S.P.: A unified stability analysis of meshless particle methods, Int. J. Numer. Meth. Engng 2000, 48, 1359-1400

[4] Belytschko T., Lu Y.Y., Gu L.: Element-free Galerkin methods, International Journal for Numerical Methods in Engineering, 37 (1994), 229-256

[5] Belytschko T., Chen H., Xu J., Zi G.: Dynamic Crack Propagation Based on Loss of Hyperbolicity and A New Discontinuous Enrichment, International Journal for Numerical Methods in Engineering, 58 (12), 1873-1905, 282003

[6] Brown K., Attaway S., Plimpton S., Hendrickson B.: Parallel strategies for crash and impact simulations, Comp. Methods Appl. Mech. Engrg. 184 (2000), 375-390

[7] Camacho G.T., Ortiz M.: Adaptive Lagrangian modelling of ballistic penetration of metallic targets, Comp. Meth. Appl. Mech. Engn. 142 (3-4), 269-301, 301997

[8] Cundall P.A., Konietzky H.: PFC-ein neues Werkzeug für numerische Modellierungen, Bautechnik 73 (1996), Heft 8

[9] Curbach M.: Festigkeitssteigerung von Beton bei hohen Belastungsgeschwindigkeiten, Institut für Massivbau und Baustofftechnologie, Universität Karlsruhe, 1987.

[10] Curran D.R., Seaman L.: Dynamic fracture criteria for ductile and brittle metals, Journal of Materials, JMLSA, Vol. 7, No. 3, 1972, 393-401 
[11] Curran D.R., Seaman L.: Dynamic failure of solids, Physics Report 147, Nos. 5 and 6 (1987), 253-388, North-Holland, Amsterdam

[12] Curran D.R., Seaman L.: Simplified models of fracture and fragmentation, Chapter 13, in: High-Pressure Shock Compression of Solids II, Dynamic fracture and fragmentation, L. Davison, D.E. Grady, M. Shahinpoor (eds.), Springer, 1993

[13] Dilts G.A.: Moving-Least-Squares-Particle Hydrodynamics I: Consistency and Stability, Int. J. Numer. Meth. Engng., 44, S.1115-1155, 1999

[14] Donze F.V. et al.: Modeling Fractures in Rock Blasting, Int. J. Rock Mech. Min. Sci., Vol. 34, No. 8, 1997, 1153-1163

[15] Eibl J., Curbach M.: Zur Steigerung der Werkstoffestigkeit bei hohen Dehngeschwindigkeiten. 13. MPA-Seminar, Universität Stuttgart, 1987

[16] Eibl J.: A constitutive Law for Concrete at fast and high Loading, Structural Dynamics-Eurodyn 2002 S.59 vol 1, 2002. Hersg: Grundmann/Schueller, A.A. Balkema Publishers Lisse/Abingdon/Exton(pa)/Tokio

[17] Gingold R.A., Monaghan J.J.: Smoothed particle hydrodynamics: theory and applications to non-spherical stars, Mon. Not. R. astr. Soc., 181, 1977, 375-389

[18] Glenn L.A., Chudnovsky A.: Strain-energy effects on dynamic fragmentation, J. Appl. Phys. 59(4), 1986

[19] Grady D.E., Kipp M.E.: Proc. 20 th Symposium on Rock Mechanics, Austin, Texas, 1979, 403

[20] Grady D.E., Kipp M.E.: Continuum modelling of Explosive Fracture in Oil Shale, Int. J. Rock mech. Min. Sci. and Geomech. Abstr., Vol.17, 1980, 147- 157

[21] Grady D.E., Kipp M.E.: Fragmentation properties of metals, Int. J. Impact Engng. Vol. 20, 1997, 293-308

[22] Grady D.E.: Local inertia effects in dynamic fragmentation, J. Appl. Phys. $53(1), 1982$

[23] Grady D.E.: Fragmentation of rapidly expanding jets and sheets, Int. J. Impact Engng. 5, 285-292, 1987

[24] Grady D.E.: The spall strength of condensed matter, J. Mech. Phys. Solids, Vol. 36, No. 3, 1988, 353-384

[25] Hanchak S.J., Forrestal M.J., Young E.R., Erhrgott J.Q.: Perforation of concrete slabs with $48 \mathrm{MPa}(7 \mathrm{ksi})$ and $140 \mathrm{MPA}(20 \mathrm{ksi})$ unconfined compressive strengths, Int. J. Impact Eng., Vol. 12, 1992, 1-7

[26] Hao S., Liu W.K., Weertman J.: Cohesive solutions of intersonic moving dislocations, PHILOS MAG 84 (11), 1067-1104, 2004

[27] Har L.A.: New Scalable Parallel Finite Element Approach for Contact Impact Problems, Ph.D. Thesis, Georgia institute of Technology, Atlanta, GA, 1998 
[28] Hermann N.: Dissertation in preparation, Institut für Massivbau, Universität Karlsruhe

[29] Hughes T.J.R., Winget J.: Finite rotation effects in numerical integration of rate constitutive equations arising in large deformation analysis, International Journal for Numerical Methods in Engineering, 1980, 15, 1862-1867

[30] Johnson G.R., Stryk R.A.: Recent Epic Code Developement for high velocity impact: 3D Element Arrangement and 2D Fragment Distribution, Int. J. Impact Engng., Vol.10, 1990, 281-294

[31] Kachanov M.: J. Eng. Mech. Division, ASCE 106 (1980), 1039

[32] Kupfer H., Hilsdorf H.K., Ruesch H., 1969. Behavior of concrete under biaxial stresses, ACI J. Vol. 66, 656-666

[33] Kipp M.E., Grady D.E.: Experimental and Numerical Studies of High-Velocity Impact Fragmentation, Chapter 12, in: High-Pressure Shock Compression of Solids II, Dynamic fracture and fragmentation, L. Davison, D.E. Grady, M. Shahinpoor (eds.), Springer, 1993

[34] Lucy L.B.: A numerical Approach to the Testing of Fission Hypothesis, Astronomical Journal, 82, 1977, 1013-1024

[35] Mills L.L, Zimmermann R.M., 1970. Compressive strength of Plain Concrete Under Multiaxial Loading Conditions, ACI Journal, Vol. 67, No. 10, Oktober, 802-807

[36] Moes N., Dolbow J., Belytschko T.: A finite element method for crack growth without remeshing, International Journal for Numerical Methods in Engineering, 46(3-4), 131-150, 1999

[37] Moes N., Gravouil A., Belytschko T.: Non-planar 3D crack growth by the extended finite element and level sets - Part I: Mechanical model, International Journal for Numerical Methods in Engineering, 53 (11), 2549-2568, 202002

[38] Morikawa H. et al.: Local fracture analysis of a reinforced concrete slab by the discrete element method, Proc. 2 nd Int. Conf. Discrete Element Meth-ods: 357-368, Cambridge, Massachusetts: IESL Publications

[39] Mott N.F., Linfoot E.H.: A theory of fragmentation, Extra-Mural Res. No. F72/80, Ministry of Supply, England, 1943

[40] Ockert J.: Ein Stoffgesetz fr die Schockwellenausbreitung in Beton, Institut für Massivbau und Baustofftechnologie, Universität Karlsruhe, 1997.

[41] Ortiz M., 1985. A constitutive theory for the inelastic behavior of concrete, Mechanics of Materials, Vol. 4, 67-93

[42] Ortiz M., Pandolfi A.: Finite-deformation irreversible cohesive elements for three-dimensional crack-propagation analysis, International Journal for Numerical Methods in Engineering, 44 (9), 1267-1282, 301999 
[43] Rabczuk T., Eibl J.: Simulation of high velocity concrete fragmentation using SPH/MLSPH, Int. J. Numer. Meth. Engng. 56, 2003, 1421-1444

[44] Rabczuk T., Eibl J.: Numerical analysis of prestressed concrete beams using a coupled Element Free Galerkin/Finite Element approach, International Journal of Solids and Structures, 41, 2004, 1061-1080

[45] Rabczuk T., Belytschko T., Xiao S.P.: Stable particle methods based on Lagrangian kernels, Comp. Meth. Appl. Mech. Eng., Vol. 193 (12-14), 1035-1063, 2004

[46] Rabczuk T., Belytschko T.: Cracking particles: A simplified meshfree method for arbitrary evolving cracks, Int. J. Numer. Meth. Engng. 61, 2004, 2316-2343

[47] Rabczuk T.: Numerische Untersuchungen zum Fragmentierungsverhalten von Beton mit Hilfe der SPH-Methode, Dissertation, 2002, Institut für Massivbau und Baustofftechnologie, Universität Karlsruhe

[48] Randles P.W., Libersky L.D.: Smoothed Particle Hydro-dynamics: Some recent improvements and applications, Comput. Methods Appl. Mech. Engrg. 139 (1996), 375-408

[49] Roos A., Chaboche J.L., Gelebart L.: Multiscale modelling of titanium aluminides, INT J PLASTICITY, 20 (4-5), 811-830, 2004

[50] Rossi P.: A physical phenomen which can explain the mechanical behavior of concrete under high strain rates, Material and Structures, 1991, 24, 422-424

[51] Xu X.P., Needleman A.: Numerical simulations of dynamic crack growth along an interface, INT J FRACTURE, 199674 (4): 289-324

[52] Yarin A.L.: Free liquid jets and films, hydrodynamics and rheology, Longman, New York, 1993, 338-348 